\title{
SYNTHESIS, CHEMICAL IDENTIFICATION, DRUG RELEASE AND DOCKING STUDIES OF THE AMLODIPINE-CHITOSAN NANOBIOPOLYMER COMPOSITE
}

\author{
RODRIGO RAMIREZ-TAGLE ${ }^{1,2}$, GUILLERMO SALGADO-MORAN ${ }^{3}$, DAVID MENDEZ-LUNA ${ }^{4}$, JOSÉ CORREA- \\ BASURTO ${ }^{4}$, WILSON CARDONA VILLADA ${ }^{5}$, LORENA GERLI CANDIA ${ }^{6}$ AND LUIS H. MENDOZA-HUIZAR ${ }^{7^{*}}$
}

\author{
${ }^{1}$ Universidad Pedro de Valdivia, Facultad de Salud. Santiago. Chile. \\ ${ }^{2}$ Quimica del Maipo Ltda, Comunidad Viña Pelvin P23 L10 Peñaflor, Chile. \\ ${ }^{3}$ Universidad de Concepcion, Investigador Extramural, Concepción, Chile. \\ ${ }^{4}$ Laboratorio de Modelado Molecular y Diseño de Fármacos, Sección de Estudios de Posgrado e Investigación, Escuela Superior de Medicina, Instituto Politécnico \\ Nacional, Mexico City, 11340, México. \\ ${ }^{5}$ Facultad de Ciencias Exactas, Departamento de Quimica, Universidad Andres Bello, Concepcion, Chile. \\ ${ }^{6}$ Departamento de Química Ambiental, Facultad de Ciencias, Universidad Católica de la Santísima Concepción, Concepción, Chile. \\ ${ }^{7}$ Autonomous University of Hidalgo State. Academic Area of Chemistry. Carreter
}

\begin{abstract}
A new amlodipine-chitosan nanocomposite was built using amlodipine nanoparticles as primary scaffolds by spontaneous emulsification, and its complete elucidation was performed by using several spectrometric techniques. Our results indicate that the amlodipine-chitosan nanocomposite has better solubility than amlodipine at $\mathrm{pH} 7.4$ with a nearly all the drug substance dissolved (97\%) by the final time-point measured. The docking study support the existence of intermolecular interactions are established between amlodipine and chitosan.
\end{abstract}

Keywords: Amlodipine; Chitosan; Nanocomposite; Drug-release properties; Docking studies.

\section{INTRODUCTION}

Water solubility is one of the most important parameters for achieving the systemic drug concentration necessary for getting a desired pharmacological response. ${ }^{1}$ The partition coefficient $(\log \mathrm{P})$ of a pharmaceutical entity is a key parameter that affects bioavailability, particularly for orally administered products. ${ }^{2}$ A poor drug bioavailability is associated with a high $\log \mathrm{P}$ value, where a high Log P value could imply a low dissolution rate in biological fluids, whereas low Log P value indicates the entity can pass through cell membranes, possibly affecting structural stability. ${ }^{3}$ Nowadays, there is a great interest in improving the physicochemical properties of pharmaceuticals with poor solubilities by employing methods such as reducing particle size, increasing surface area, coupling or coating with other entities among others. ${ }^{4-15}$ These procedures allow to increase the ability of these drugs to dissolve in an aqueous medium and thus meeting required therapeutic concentrations. ${ }^{4-15}$

Amlodipine besylate (2-[(2-aminoethoxy)-methyl]- 4-(2-chlorophenyl) 1, 4dihydro-6-methyl-3,5-pyridine-dicarboxylic acid-3 ethyl-5 methyl ester; Fig. 1) is a calcium channel blocker with extremely potent vasodilating activity, used for the management of hypertension, angina pectoris, and cerebrovascular disease. ${ }^{16-17}$ However, it has low water solubility in the $\mathrm{pH}$ range from 1 to 6.8 . In this $\mathrm{pH}$ range at $37^{\circ} \mathrm{C}$, water solubility is $1 \mathrm{mg} / \mathrm{mL} .{ }^{18} \mathrm{Here}$, it is convenient to mention that nanoemulsions are able to improve the oral bioavailability and subsequent release of some drugs. These nanoemulsions can be built using polymers or lipidmaking nanoparticles to develop therapeutic and pharmacological properties. ${ }^{19}$

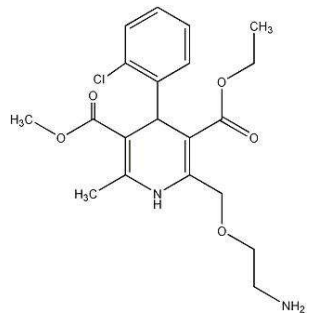

Figure 1. Molecular structure of amlodipine

Chitosan is a biopolymer built of $\beta$-(1,4)-linked glucosamine elements. ${ }^{20}$ It exhibits mucoadhesive properties because of its positive charge at the protonated amine terminal, favoring electrostatic interaction with the negatively charged mucosal surface, allowing easier transport across transmucosal barriers. It is known that chitosan is biodegradable and biocompatible and has low toxicity properties, making it appropriate for use in pharmaceutical and biomedical investigations. ${ }^{21-23}$ Also, it is well known that chitosan can control drug release and the oral bioavailability of amlodipine. ${ }^{24-27}$
Thus, it is possible to suggest that the formation of an amlodipine-chitosan complex could improve the water solubility and oral bioavailability of amlodipine. In this sense, the main aim of this study is to carry out the synthesis and characterization of a new amlodipine-chitosan complex and to demonstrate that it presents a higher solubility in water due to the formation of the nanocomposite. In addition, we perform docking studies to analyze the key interactions established between amlodipine and chitosan that evidence the formation of nanoparticles and explain how these chemical interactions improve the amlodipine release.

\section{EXPERIMENTAL DETAILS}

\subsection{Material and methods}

Amlodipine was obtained from Alborz Darou. Chitosan purchased from Sigma-Aldrich with medium molecular weight $(75-85 \%$ deacetylated). The chemical grade, laboratory vendor or supplier, and most importantly, the molecular size (in $\mathrm{kDa}$ ) and deacetylation percentage are of concern. A multiwave ultrasonic generator (Sonicator_3000; Misonix, Inc., Farmingdale, NY, USA) equipped with a converter/transducer and titanium oscillator (horn), 12.5 $\mathrm{mm}$ in diameter, was operated at $20 \mathrm{kHz}$ with a maximum power output of 600 W. X-ray powder diffraction (XRPD) measurements were performed using an X'pert Diffractometer (Philips; United States) with monochromated $\mathrm{CuKa}$ radiation. The Fourier transform-infrared (FT-IR) spectra between 400 and 4000 $\mathrm{cm}^{-1}$ were recorded using a Bruker spectrometer and $\mathrm{KBr}$ pellets. The UV-Vis spectra was obtained by UV-Vis Spectrophotometer, PhotonixAr 2017, Iran. The morphology of both the nanoparticle and nanocomposite preparations were studied using scanning electron microscopy (SEM; LEO 1430VP) and the gold sputtering technique.

\subsection{Preparation of amlodipine nanoparticles}

$1 \mathrm{~g}$ of amlodipine was dispersed in $30 \mathrm{~mL}$ of acetonitrile and sonicated at 90 $\mathrm{W}$ for $20 \mathrm{~min}$ with a high-density ultrasonic probe immersed directly in the dispersion. This method has proved to be an adequate strategy for dispersing materials during the synthesis of nanoparticles in a liquid phase ${ }^{28}$. Then the amlodipine was separated from the acetonitrile by centrifugation $(6,000 \mathrm{rpm}, 1$ $\mathrm{min})$. The product was dried at room temperature under vacuum.

\subsection{Preparation of amlodipine-chitosan nanocomposite}

The nanocomposite was prepared using the spontaneous emulsification method. The solution was prepared by dissolving $0.15 \mathrm{~g}$ of chitosan in $50 \mathrm{~mL}$ of distilled water, slightly acidified with $0.5 \mathrm{~mL}$ of acetic acid. It was stirred for 2 $\mathrm{hr}$ at $40^{\circ} \mathrm{C}$. This solution was added dropwise to a suspension containing $0.07 \mathrm{~g}$ of amlodipine dissolved in $30 \mathrm{~mL}$ of distilled water, and this solution was stirred 
for $24 \mathrm{hr}$ at $40^{\circ} \mathrm{C}$. The solids were filtered, and washed with deionized water, and then dried at $80^{\circ} \mathrm{C}$.

\subsection{In vitro drug release properties}

The release rate of amlodipine from the amlodipine-chitosan nanocomposite was measured at $\mathrm{pH} 7.4$ (phosphate buffered saline). In-vitro drug release studies were carried out using a pore size based on the molecular size of amlodipine. To this end, $0.005 \mathrm{~g}$ nanocomposite and $5 \mathrm{~mL}$ buffer were transferred to a porous dialysis membrane, and the tube was placed in $500 \mathrm{~mL}$ of the same release medium at $37^{\circ} \mathrm{C}$. It was stirred at $100 \mathrm{rpm}$ for $24 \mathrm{~h}$. The solution was filtered through a $0.45-\mu \mathrm{m}$ filter. Every $5 \mathrm{~min}, 3 \mathrm{~mL}$ of the solution was removed and immediately replaced by an equal volume of fresh buffer (to keep the volume of diffusion medium constant). The amount of drug released was monitored by using UV-Vis spectrophotometry at $257 \mathrm{~nm}$. The percent of drug released was evaluated using the following formula:

$$
\text { Drug Release }(\%)=\frac{\text { Amount of drug release }(\mathrm{mg})}{\text { Total weight of drug sample }(\mathrm{mg})} \times 100
$$

\subsection{Docking studies}

In order to analyze the amlodipine-chitosan interaction properties, docking studies were performed following a well-established method of elucidating the binding mode and how these interactions affect the releasing properties. In the first stage, the molecular structures of amlodipine and chitosan were built using the ChemSketch program ${ }^{29}$ then they were minimized using the semi-empirical AM1 method as implemented in the Gaussian 03 software. ${ }^{30}$ The optimized structures were prepared for the docking study through Gaussview $05^{31}$. AutoDock Tools was used as the graphical interface, and all docking simulations were performed using AutoDock 4.0, ${ }^{32}$ applying the Genetic Lamarckian Algorithm to obtain the best-ranked binding modes selected by the energetically most stable criteria, measured by the binding energy $(\Delta G, \mathrm{kcal} / \mathrm{mol})$. Briefly, the docking procedure consisted of covering the entire amlodipine-chitosan nanocomposite with a $60 \times 60 \times 60 \AA^{3}$ grid at a grid point spacing of $0.375 \mathrm{~A}^{3}$. Energy evaluations were completed using $1 \times 10^{7}$ runs, and the outcome topologies were analyzed using Pymol graphical viewer. ${ }^{33}$

\section{RESULTS}

\subsection{X-ray powder diffraction analysis}

X-ray powder diffraction (XRPD) of amlodipine was first reported by Koradia et al. in 2010. ${ }^{34-35}$. Upon comparing our XRPD studies with those reported elsewhere, we found very similar diffraction patterns. The structure and particle size of amlodipine, found using XRPD, is shown in Fig. 2. The average particle size was found to be about 50 to $70 \mathrm{~nm}$ when the Scherrer's equation (1) was used:

$$
\mathrm{D}=\mathrm{K} \lambda /(\beta \cos \theta)
$$

where $\mathrm{D}$ is the mean crystalline size (nm), $\mathrm{K}$ is 0.9 (Scherrer's constant), $\lambda$ is $0.154 \mathrm{~nm}, \beta$ is a half band width, and $\theta$ is the peak angle. ${ }^{36}$ The XRPD pattern of a typical sample prepared by the sonochemical process was obtained, and the Sherrer formula was used to estimate the particle size from the broadening of the XRPD peaks. The average particle size was found, and it coincided with that found using SEM images. ${ }^{37-40}$

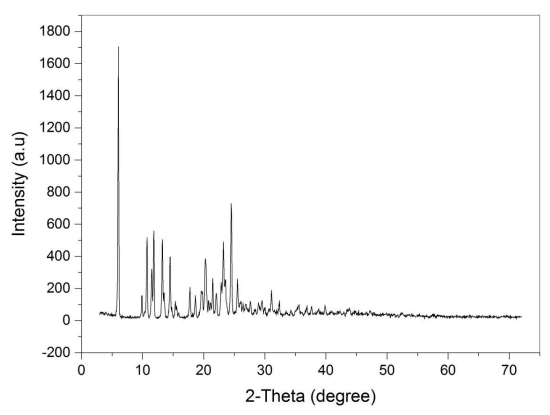

Figure 2. X-ray powder diffraction pattern of amlodipine nanoparticles prepared by a sonochemical process.

\subsection{Vibration spectra}

Amlodipine nanoparticles and the chitosan/amlodipine-chitosan nanocomposite were analyzed by FT-IR, and the resultant spectra are shown in Fig. 3 parts A and B, respectively. The FT-IR spectrum of pure amlodipine was reported by Szabó et al., ${ }^{41}$ and our findings agree, showing a peak at $3301 \mathrm{~cm}^{-1}$ representing N-H asymmetrical stretching and one at $3160 \mathrm{~cm}^{-1}$ representing N$\mathrm{H}$ stretching in the secondary amine. The bending deformation of $\mathrm{NH}_{2}$ was found at $1495 \mathrm{~cm}^{-1}$. The peaks at 1698,1675 , and $1617 \mathrm{~cm}^{-1}$ resulted from $\mathrm{C}=\mathrm{O}, \mathrm{NH}_{3}$, and carboxylate $\mathrm{C}=\mathrm{C}$ stretching, respectively. At 2982 and $2949 \mathrm{~cm}^{-1}$, stretching vibrations of $\mathrm{CH}_{3}$ and $\mathrm{NH}_{3}$ were seen, and at $1017 \mathrm{~cm}^{-1}$ stretching vibrations of $\mathrm{C}-\mathrm{H}$ and the C-C-C ring were seen. The peaks at 1303 and $1093 \mathrm{~cm}^{-1}$ were assigned to $\mathrm{C}-\mathrm{H}$ and $\mathrm{SO}_{3}$ stretching vibrations; the one at $1206 \mathrm{~cm}^{-1}$ was assigned to $\mathrm{SO}_{3}$ and $\mathrm{C}-\mathrm{C}$ stretching, $\mathrm{C}-\mathrm{H}$ bending, and $\mathrm{NH}_{3}$ rocking vibrations. Finally, at $755 \mathrm{~cm}^{-1}, \mathrm{C}-\mathrm{H}$ ring vibrations were seen. These results proved the purity and accuracy of the amlodipine nanoparticles prepared ultrasonically and the amlodipine-chitosan nanocomposite.

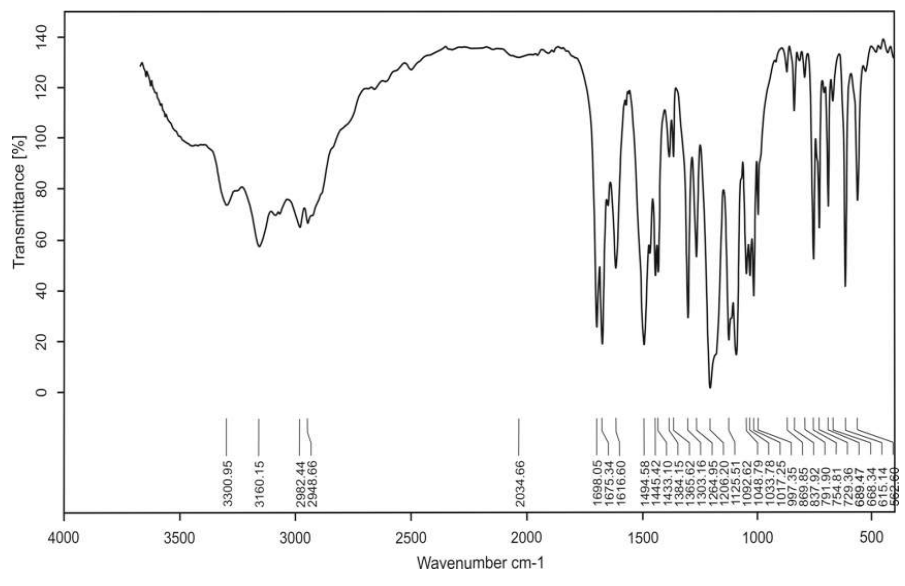

(A)

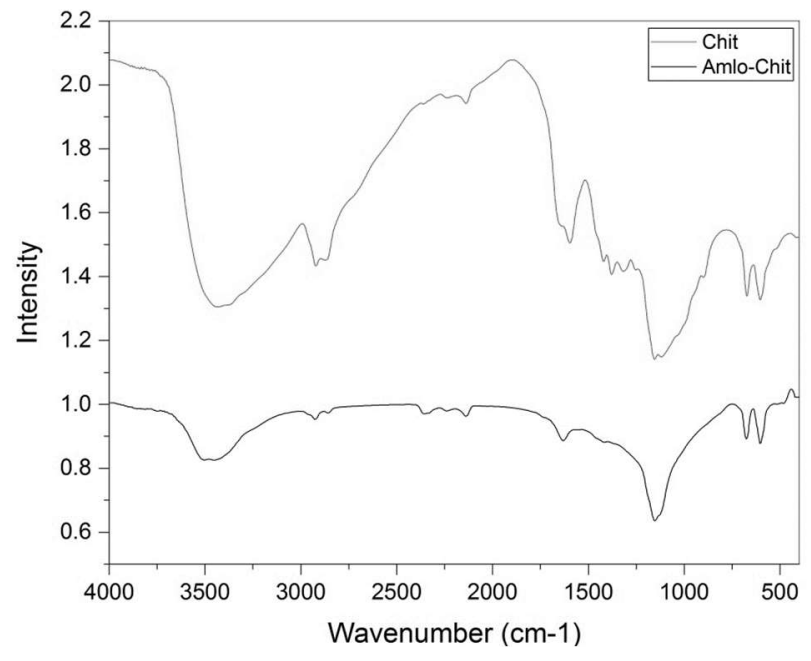

(B)

Figure 3. Fourier transform-infrared spectra: (A) amlodipine nanoparticles, (B) chitosan (red spectrum) amlodipine-chitosan nanocomposite (black spectrum) in bulk $\mathrm{KBr}$.

\subsection{Morphological properties}

The morphology, structure, and size of the amlodipine nanoparticles and amlodipine-chitosan nanocomposite (Figs. 4 and 5, respectively) were investigated by SEM. The images obtained for amlodipine nanoparticles depicted an amorphous morphology and a particle size of about 50 to $70 \mathrm{~nm}$ (Fig. 4). Those obtained for the amlodipine-chitosan nanocomposite showed very good homogeneity without an amorphous phase and showed that good quality was achieved in its preparation (Fig. 5). 

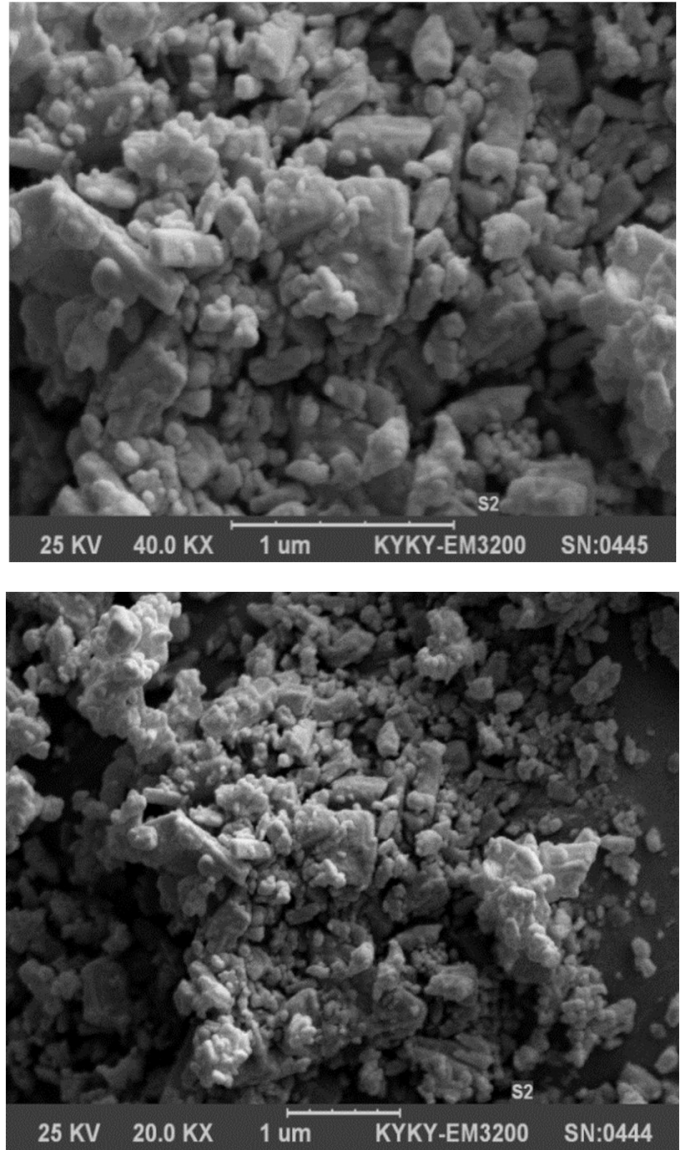

Figure 4. Scanning electron microscopy images of amlodipine nanoparticles. Left, $40 \mathrm{KX}$; right, $20 \mathrm{KX}$.
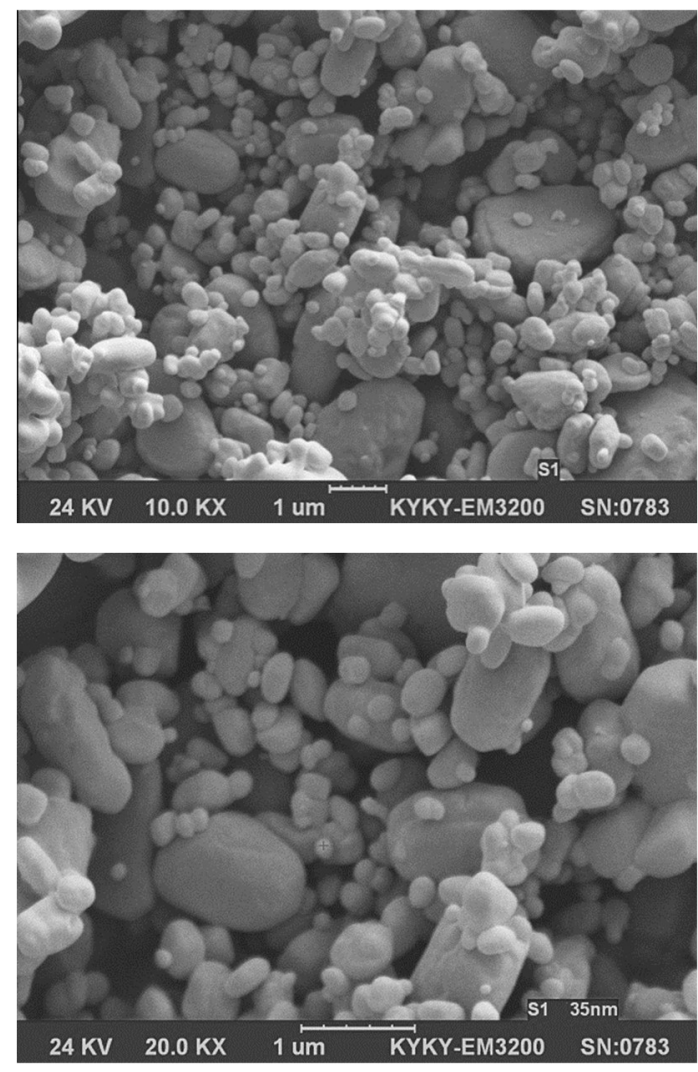

Figure 5. Scanning electron microscopy images. Amlo-Chitosan nanocomposite. Left, $10 \mathrm{KX}$; right, $20 \mathrm{KX}$.

\section{DISCUSSION}

\subsection{Drug release studies}

Anionic polymers form a matrix system that can be used as a controlled drug release method. ${ }^{42-43}$ Drug release studies are critical for determining the behavior that a drug might exhibit in the human body. The release rate of amlodipine from the amlodipine-chitosan nanocomposite, measured at $\mathrm{pH} 7.4$ (phosphate buffered saline), is shown in Fig. 6 . The quantity of drug released was monitored using UV-Vis spectrophotometry at $257 \mathrm{~nm}$. From Fig. 6, note that the amlodipinechitosan nanocomposite shows a greater release rate in comparison to release rate associated with amlodipine. Maximum release rates of $97 \%$ and $66 \%$ for amlodipine-chitosan and amlodipine, respectively, were found at $25 \mathrm{~min}$, the time point limit, demonstrating that the amlodipine nanocomposite had a better release at $\mathrm{pH} 7.4 .44-45$

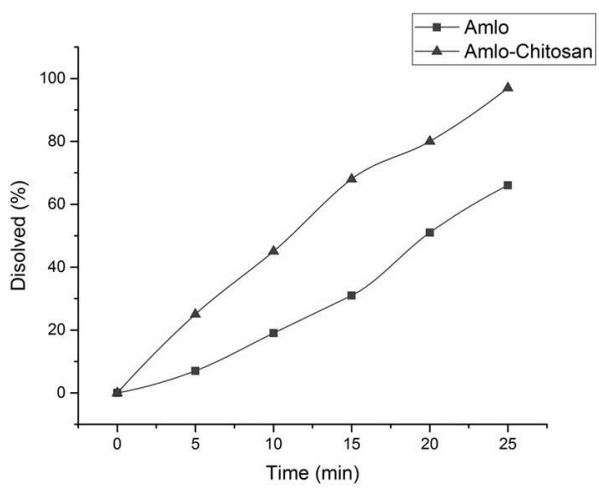

Figure 6. Rate of solubility of amlodipine and amlodipine-chitosan nanocomposite in water at $\mathrm{pH}=7.4$.

\subsection{Docking analysis.}

Because the chitosan structure includes both donor and acceptor hydrogen atoms, it was highly expected that the binding modes established between it and amlodipine were stabilized primarily by hydrogen bonds. Indeed, the preferred binding mode of amlodipine docked over chitosan shows 2 hydrogen bonds at $<2.5 \AA$, and a special orientation free of steric hindrance led to an improved energy state where $\Delta G$ for $\mathrm{A}, \mathrm{B}, \mathrm{C}$, and $\mathrm{D}$ were $-1.72,-1.21,-1.2$, and -1.1 $\mathrm{kcal} / \mathrm{mol}$, respectively (Fig. 7A). The same behavior was shown by the bestranked binding modes, where hydrogen bond formation decreased, but energy remained good (Figs. 7B, C, and D).
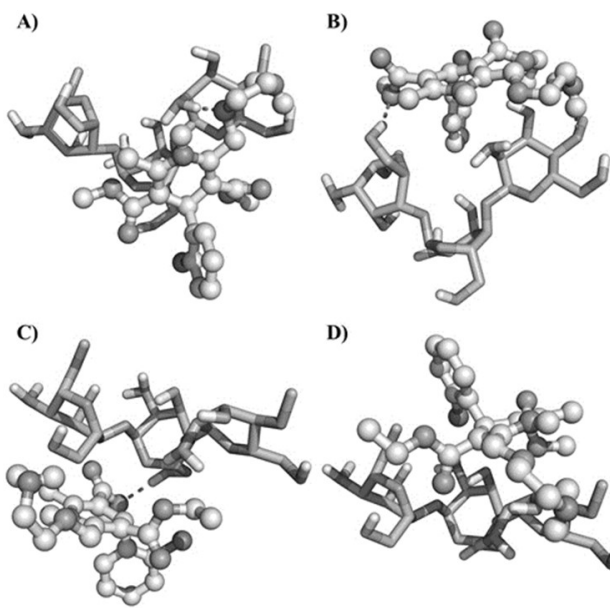

D)

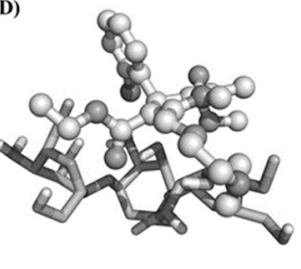

Figure 7. Best-ranked binding modes yielded by the docking study. Cyan dashes represent hydrogen bonds.

Here, it is important to mention that it is necessary to conduct further analyses as organ-isolated studies so that the amlodipine-chitosan nanocomposite and amlodipine can be compared in their abilities to generate the pharmacological effect required. However, such study is beyond of the scope of the present work. 


\section{CONCLUSIONS}

A new amlodipine-chitosan nanocomposite was built by using amlodipine nanoparticles as primary scaffolds by mean spontaneous emulsification. Data obtained show that the amlodipine-chitosan nanocomposite has a better solubility than amlodipine at $\mathrm{pH} 7.4(97 \%)$. The docking study reveals that hydrogen bonds are primarily responsible for enhancing the solubility of the nanocomposite.

\section{CONFLICT OF INTEREST}

The authors have no conflicts of interest to declare.

\section{ACKNOWLEDGMENTS}

We gratefully acknowledge financial support from the Research Council of Imam Khomeini International University and Universidad Pedro de Valdivia.

\section{REFERENCES}

1. V. R. Vemula, V. Lagishetty, and S. Lingala, Int J Pharm Sci Rev Res. (2010), $5(1), 41$.

2. S.N. Kokil, PhD Dissertation, Long Island University, THE BROOKLYN CENTER, 2009, 215 pages; $3371316 \quad$ (2009). http://search.proquest.com/docview/304994657

3. D.M. Brahmankar, and S.B. Jaiswal, 1st ed. Delhi: Vallabh Prakashan, (2006), 296.

4. S.G. Kapsi, and J.W. Ayres, Int J Pharm. (2001), 23(229), 193-203.

5. Xi. Han, C. Ghoroi, D. To, Y. Chen, and R. Davé, Int J Pharm. (2011), 415, 185-195.

6. G.G. Liversidge, and K.C. Cundy, Int J Pharm. (1995), 125, 91-97.

7. R.H. Müller, K. Peters, Int J Pharm. (1998), 160, 229-237.

8. S. Ghanbarzadeh, H. Valizadeh, and P. Zakeri-Milani, Adv Pharm Bull. (2013), 3, 25-29.

9. S.F. Wang, F. Gu, and M.K. Lu, Langmuir (2006), 22: 398

10. J.Z. Xu, S. Xu, J. Geng, G.X. Li, and J.J. Zhu, Ultrason Sonochem. (2006), $13,451-454$.

11. S.Y. Zhang, Y. Liu, X. Ma, and H.Y. Chen, J Phys Chem B. (2006), 110(18), 9041-9047.

12. H. Lei, Y.J. Tang, J.J. Wei, J. Li, X.B. Li, and H.L. Shi, Ultrason Sonochem. (2007), 14(1): 81-83.

13. V.G. Kumar, and K.B. Kim, Ultrason Sonochem. (2006), 13(6): 549-556.

14. C.J. Mao, H.C. Pan, X.C. Wu, J.J. Zhu, and H.Y. Chen, J Phys Chem B. (2006), 110(30), 14709-14713.

15. A. Lashgari, S. Ghamami, S. Shahbazkhany, G. Salgado-Morán, and D. Glossman-Mitnik, $J$ Nanomater. Article ID 384835 (2015). Doi: $10.1155 / 2015 / 384835$.

16. V.G. Dongre, S.B. Shah, P.P. Karmuse, M. Phadke, and V.K. Jadhav, $J$ Pharm Biomed Anal. (2008), 46, 583e586.

17. A.K. Sarkar, D. Ghosh, A. Das, P.S. Selvan, K.V. Gowda, U. Mandal, A. Bose, S. Agarwal, U. Bhaumik, and T.K. Pal, .J Chromatogr B. (2008), 873(1):77-85.

18. WHO Expert Committee on Specifications for Pharmaceutical Preparations WHO Technical Report Series, No. 992 - Forty-ninth Report (Geneva, 1317 October 2014)

19. T.M. Allen, and P.R. Cullis, Science (2004), 303: 1818-1822.

20. I. Aranaz, M. Mengíbar, R. Harris, I. Panos, B. Miralles, N. Acosta, G. Galed, and A. Heras, Curr Chem Biol. (2009), 3(2):203-230.

21. Y. Shi, A. Wan, Y. Shi, Y. Zhang, and Y. Chen, Biomed Res Int. (2014), Article ID 613619. doi: 10.1155/2014/613619

22. O. Felt, P. Buri, and R. Gurny, Drug Dev Ind Pharm. (1998), 24:979-993.

23. A. Di Martino, M. Sittinger, and M.V. Risbud, Biomaterials, (2005), 26:5983-5990.

24. W.E. Rudzinski, and T.M. Aminabhavi, Int J Pharm. (2010), 399:1-11.

25. S.A. Agnihotri, and T.M. Aminabhavi, Drug Dev Ind Pharm. (2007), 33:1254-1262.

26. S.C. Angadi, L.S. Manjeshwar, and R.M. Aminabhavi, Ind Eng Chem Res. (2013), 52: 6399-6409.

27. G. Chhabra, K. Chuttani, A.K. Mishra, K. Pathak, Drug Dev Ind Pharm. (2011), 37:907-916.

28. J.S. Taurozzi, V.A Hackley, M.R. Wiesner. Nanotoxicology (2010), 5(4), 711-729.

29. ACD/Structure Elucidator, version 15.01 [Internet]. Toronto (ON): Advanced Chemistry Development, Inc. c1996-2015- www.acdlabs.com

30. M.J. Frisch, G.W. Trucks, H.B. Schlegel, G.E. Scuseria, M.A. Robb, J.R. Cheeseman, and et al Gaussian 98 Revision A.7. Gaussian Inc, Pittsburgh, P.A, 1998.
31. R. Dennington, T. Keith and J. Millam, “Gauss View Version 5," Semichem Inc., Shawnee Mission, 2009.

32. G.M. Morris, R. Huey, W. Lindstrom, M.F. Sanner, R.K. Belew, D.S. Goodsell, and et al. J. Comput. Chem. (2009), 30:2785-2791.

33. L.L.C. Schrödinger, The PyMOL Molecular Graphics System [type of medium]. Version 1.7.4. 2019

34. V. Koradia, H.L. de Diego, K. Frydenvang, M. Ringkjøbing-Elema, A. Mullertz, A.D. Bond, and et al. Cryst Growth Des., (2010), 10(12):52795290 .

35. V. Koradia, A.F. de Lemos, M. Allesø, H.L. d e Diego, M.R. RingkjøbingElema, A. Mullertz, and et al., J Pharm Sci. (2011), 100:2896-2910.

36. A. Lashgari, S. Ghamami, Z.Bahrami, F. Shomossi, G. Salgado-Morán, and D. Glossman-Mitnik, J Nanomater., Article ID 130698, 2015. doi:10.1155/2015/130698.

37. S. Ghamami, M. Golzani, and A. Lashgari, J. Chil. Chem. Soc. (2016), 61(2):2954-2957.

38. A. Lashgari, S. Ghammamy, L. Gerli, and G. Salgado Morán, Advances in Environmental Biology, (2014), 8(21):339-345.

39. S. Ghamami, S. Kazemzade Anari, M. Bakhshi, A. Lashgari, G. SalgadoMorán, and D. Glossman-Mitnik, Open Chem. (2016), 14, 60-64.

40. A. Lashgari, S. Ghammami, M. Golzani, L. Gerli-Candia, G. Salgado-Morán, D. Glossman-Mitnik, and B. Abdolmaleki, J. Chil. Chem. Soc. (2016), 61(4), 3201-3205.

41. L. Szabó, V. Chiş, A. Pîrnău, Leopold N., Cozar O., and Orosz S. J. Mol. Struct., (2009), 924-926: 385-392.

42. Y. Shao, L. Li, X. Gu, L. Wang, and S. Mao, Asian J Pharm Sci. (2015), 10:24-30

43. E. Ramírez-San Juan, M.A. Soriano-Ursúa, J.E. Raya, J. Correa-Basurto, J.G. Trujillo-Ferrara, R. Miranda-Ruvalcaba, and et al., Med Chem Res. (2014), 32(12): 5149-5159.

44. S. Ghamami, M. Golzani, and A. Lashgari, J Incl Phenom Macrocycl Chem. (2016), 86(1): 67-78.

45. S. Ghamami, A. Lashgari, and M. Golzani, J Incl Phenom Macrocycl Chem. (2017), 88(1):1-14. 